\title{
MONITORANDO A DEGLUTIÇÃO ATRAVÉS DA ELETROMIOGRAFIA DE SUPERFÍCIE
}

\section{Monitoring swallowing with surface electromyography}

\author{
Maria das Graças Wanderley de Sales Coriolano (1), Otávio Gomes Lins ${ }^{(2)}$, \\ Luciana Rodrigues Belo ${ }^{(3)}$, Danielle Carneiro de Menezes ${ }^{(4)}$, Silvia Regina Arruda Moraes ${ }^{(5)}$, \\ Amdore Guescel Asano ${ }^{(6)}$, Paulo José de Andrade Lira Oliveira ${ }^{(7)}$, Douglas Monteiro da Silva ${ }^{(8)}$
}

\begin{abstract}
RESUMO
Objetivo: descrever o método de registro da eletromiografia de superfície através da utilização de um protocolo desenvolvido para o estudo da deglutição e demonstrar a deglutição de um paciente com doença de Parkinson e de um sujeito normal através do registro da eletromiografia de superfície (EMGs). Métodos: para ilustrar os parâmetros eletrofisiológicos registrados após execução do protocolo foram utilizados dois voluntários do sexo feminino, sendo um sem doença e outro apresentando doença de Parkinson (DP) no estágio III de acordo com a escala de Hoehn e Yahr. Os parâmetros analisados pelo foram: a duração da atividade elétrica durante a deglutição, a amplitude (rms) e o limite de disfagia. Resultados: os resultados mostram diferenças entre os eletromiogramas ilustrativos. Conclusão: a EMGs pode ser utilizada como método de avaliação e monitorização da deglutição de sujeitos sem doença e com DP.
\end{abstract}

DESCRITORES: Deglutição; Transtornos da Deglutição; Neurofisiologia; Eletromiografia

(1) Fisioterapeuta; Professora Assistente da Universidade Federal da Paraíba, UFPB, João Pessoa, PB, Brasil; Doutoranda do Departamento de Neuropsiquiatria e Ciências do Comportamento da Universidade Federal de Pernambuco.

(2) Médico Neurologista; Professor Adjunto da Universidade Federal de Pernambuco UFPE, Recife, PE, Brasil; PhD em Neurologia/Neurociências pela Universidade Federal de São Paulo.

(3) Fonoaudióloga do Centro Médico Odontológico, Recife, PE, Brasil; Especialista em Motricidade Orofacial pelo CEFAC - Pós-Graduação em Saúde e Educação; Mestranda do Departamento de Pós-Graduação em Neuropsiquiatria e Ciências do Comportamento da Universidade Federal de Pernambuco.

(4) Terapeuta Ocupacional; Professora Substituta da Universidade Federal de Pernambuco UFPE, Recife, PE, Brasil; Mestranda do Departamento de Pós-Graduação em Neuropsiquiatria e Ciências do Comportamento da Universidade Federal de Pernambuco.

(5) Fisioterapeuta; Professora Adjunta da Universidade Federal de Pernambuco UFPE, Recife, PE, Brasil; Doutora em Ciências Morfofuncionais pela Universidade de São Paulo.

(6) Médico Neurologista do Serviço de Atenção ao Doente de Parkinson do Hospital das Clínicas de Pernambuco, HC, Recife, PE, Brasil; Mestre Neuropsiquiatria e Ciências do Comportamento pela Universidade Federal de Pernambuco.

(7) Aluno do curso de Graduação em Fisioterapia da Faculdade Maurício de Nassau, Recife, PE, Brasil; Aluno Voluntário do Programa Institucional de Bolsas de Iniciação Científica, PIBIC.

\section{INTRODUÇÃO}

A deglutição normal é uma atividade neuromuscular complexa e dinâmica que depende de um jogo de comportamentos fisiológicos que resultem no movimento eficiente e seguro de líquidos e sólidos da boca até o estômago ${ }^{1}$.

$\mathrm{O}$ ato da deglutição pode ser dividido nas fases oral, faríngea e esofágica. A fase oral, inicial, é principalmente voluntária. A fase faríngea consiste em diversas ações coordenadas para transportar o alimento da orofaringe para o esôfago, sem aspiração. Frequentemente as fases oral e faríngea são descritas juntas, e referidas como fase orofaríngea da deglutição ${ }^{2}$. Uma vez acionada, a fase faríngea é involuntária e automática, enquanto que a fase esofágica é involuntária e está controlada pelo sistema nervoso autônomo ${ }^{3}$.

A disfagia é caracterizada por uma alteração que ocorre no processo da deglutição, resultando num

(8) Aluno do curso de Graduação em Fisioterapia da Faculdade Maurício de Nassau, Recife, PE, Brasil; Aluno Voluntário do Serviço de Assistência ao Doente de Parkinson do Hospital das Clínicas de Pernambuco.

Conflito de interesses: inexistente 
redirecionamento do bolo na cavidade oral para as cavidades nasal ou laríngea ${ }^{4}$.

Entre os exames instrumentais utilizados na avaliação da disfagia, a videofluoroscopia é considerada o método-ouro na investigação de sua natureza e extensão ${ }^{5,6}$. No entanto, esse método é caro, consome tempo, expõe o paciente à radiação e, muitas vezes, somente é realizado em clínicas radiológicas especializadas ${ }^{2}$.

Além disso, falta a videofluoroscopia a mesma definição temporal do estudo eletrofisiológico para avaliar o sincronismo de cada evento funcional da deglutição ?

Já a eletromiografia de superfície (EMGs), que pode facilmente ser realizada em um laboratório padrão de eletromiografia (EMG), constitui um método não invasivo, simples, reprodutível, de baixo nível de desconforto durante o exame e que pode fornecer dados importantes para a avaliação de certos parâmetros da deglutição e contribuir para uma melhor compreensão do mecanismo fisiológico da deglutição normal e patológica ${ }^{2,8}$.

Entretanto, poucos estudos têm relatado os padrões da atividade eletromiográfica (EMG) dos músculos envolvidos na sequência da deglutição ${ }^{9}$. Normalmente, a maioria dos autores realiza o registro da atividade eletromiográfica apenas do grupo muscular supra-hióideo para medir o início e a duração da deglutição ${ }^{7,9-12}$.

É importante comentar que a EMGs não substitui a videofluoroscopia na avaliação da deglutição. $\mathrm{Na}$ verdade, esses exames são complementares para o estudo e diagnóstico dos distúrbios da deglutição ${ }^{7,13}$.

O objetivo deste trabalho é descrever o método de registro da eletromiografia de superfície através da utilização de um protocolo desenvolvido para o estudo da deglutição e demonstrar a deglutição de um paciente com doença de Parkinson e de um sujeito normal através da comparação do registro da EMGs.

\section{MÉTODOS}

Trata-se de um estudo observacional de corte transversal que foi conduzido conforme os princípios da declaração de Helsinki. O protocolo desenvolvido para registro através da eletromiografia de superfície (EMGs) foi testado com dois sujeitos do sexo feminino: Al, 63 anos, portadora da doença de Parkinson (Hoehn Yarh = 3), no período "off", ou seja, antes de tomar a medicação anti-parkinsoniana; e MGM, 60 anos, sem a doença. Al, com doença de Parkinson (DP) e MGM, normal (N), ambas apresentaram consentimento livre e esclarecido. O registro ocorreu no mês de abril de 2009 no Laboratório de Eletroneuromiografia do Hospital das Clínicas de Pernambuco.

Para a realização do registro foi utilizado um aparelho de EMGs, modelo EMG 400c, com 4 canais da marca EMG System do Brasil LTDA. A captação da atividade elétrica foi realizada por meio de eletrodos auto-adesivos descartáveis (Meditrace 200).

Antes da fixação dos eletrodos, a pele foi limpa com gaze embebida por álcool à $70^{\circ}$ e levemente abrasada com lã de aço. O eletrodo terra foi fixado sobre a clavícula direita. A mesa onde o equipamento foi instalado, assim como as cadeiras utilizadas pelos examinadores e voluntários era de madeira. Os demais utensílios, como copos e seringa, eram de plástico.

O sinal captado pelos eletrodos foi amplificado 2000 vezes (rejeição de modo comum $>120 \mathrm{~dB}$ ), filtrado com filtro passa-banda de 20 a $500 \mathrm{~Hz}$ e digitalizado a uma velocidade de $8 \mathrm{KHz}(2 \mathrm{KHz}$ por canal, 4 pontos por ciclo da frequência máxima presente no sinal).

Os músculos e grupos musculares registrados foram (Figura 1):

a) ORBICULAR DA BOCA (canal 1): dois eletrodos foram fixados lateralmente aos ângulos externos da boca. Os eletrodos foram cortados de modo a não atrapalhar o movimento dos lábios.

b) MASSETER (canal 2): dois eletrodos foram fixados nas margens inferiores do corpo da mandíbula, próximo ao seu ângulo. O local exato foi obtido pedindo-se ao sujeito para apertar os dentes e palpando-se o ventre muscular de cada masseter.

c) Grupo de músculos supra-hióideos (canal 3): esse grupo de músculos está formado pelos músculos: milohióideo, geniohióideo e ventre anterior do digástrico. Os dois eletrodos foram fixados entre o queixo e o osso hioide, um de cada lado. O centro de um eletrodo ficou separado $2 \mathrm{~cm}$ do centro do outro eletrodo.

d) Grupo de músculos infra-hióideos (canal 4): esse grupo de músculos está formado pelos músculos: esternohióideo, esternotireóideo, tíreo-hióideo e ventre superior do omo-hióideo. Os dois eletrodos foram fixados região anterior direita do pescoço, lateralmente à cartilagem tireóide da laringe, verticalmente separados 2 $\mathrm{cm}$ um do outro (centro a centro). Os eletrodos foram cortados, de modo a não atrapalhar o movimento da laringe.

O protocolo de registro foi desenvolvido com base nos estudos de Vaiman $\mathrm{M}^{8}$ e Ertekin $\mathrm{C}^{13}$. $\mathrm{O}$ registro da deglutição foi feito em duas condições: deglutição de uma só vez de um volume colocado à boca e deglutição livre de água. 
A

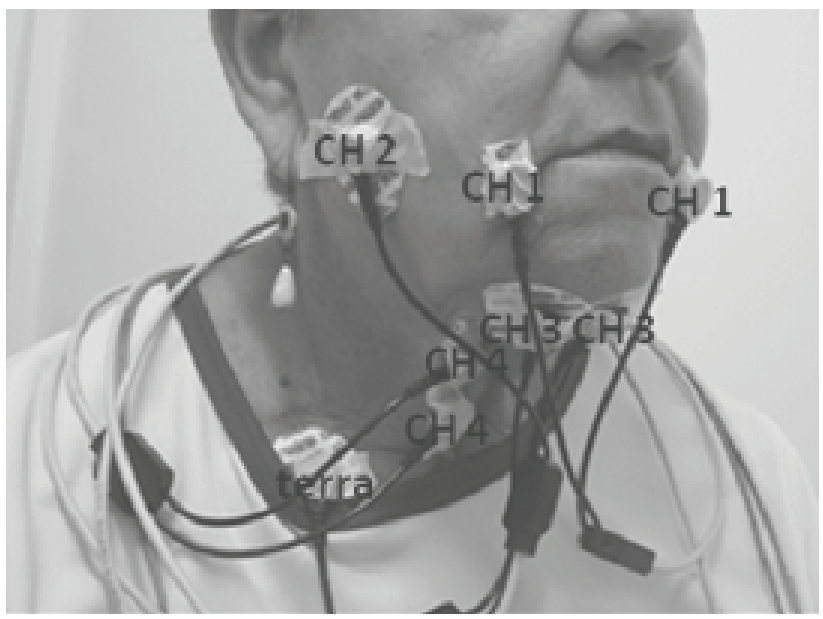

B

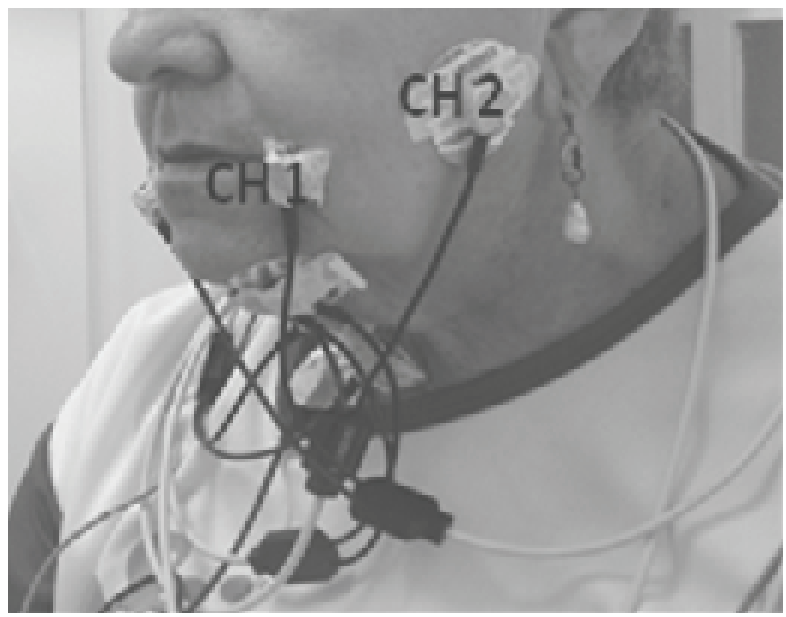

Figura 1 - Posicionamento dos eletrodos de EMGs em um voluntário representativo. A Figura 1 A ilustra o lado direito da face; a Figura $1 \mathrm{~B}$ ilustra o lado esquerdo da face. $\mathrm{CH}=$ canal.

1a condição - deglutição de uma só vez de um volume colocado à boca: Nesta condição foram utilizadas duas consistências: (1) água e (2) iogurte de consistência firme. Para a água foram utilizados dois volumes, $10 \mathrm{ml}$ e $20 \mathrm{ml}$, e para o iogurte foram utilizados também 2 volumes, $5 \mathrm{ml}$ e $10 \mathrm{ml}$. Os volumes de água e de iogurte foram medidos e colocados na boca do sujeito através de uma seringa plástica descartável. O sujeito foi orientado a manter o conteúdo na boca, engolindo somente após o comando do pesquisador.

$2^{\text {a }}$ condição - deglutição livre de água: Nesta condição foi oferecido ao sujeito um copo plástico descartável com $100 \mathrm{ml}$ de água. O sujeito foi orientado a beber normalmente após o comando. Após 2 segundos de registro da EMGs o pesquisador anunciava o comando para engolir.

\section{Processamento e análise dos sinais}

As medidas de duração e amplitude RMS (root mean square = raiz média quadrática) da atividade eletromiográfica durante as deglutições foram feitas utilizando funções próprias do EMG System.

O início e o final das deglutições foram marcados levando em consideração a atividade eletromiográfica de base que precedia e sucedia a deglutição. $O$ início da deglutição era considerado quando a atividade eletromiográfica elevava-se claramente acima da atividade de base precedente. $O$ final da deglutição era marcado quando a atividade eletromiográfica retornava aos níveis da atividade de base. A diferença entre o final da deglutição e o início da deglutição determina a duração da atividade eletromiográfica durante a deglutição. No caso da ocorrência de duas ou mais deglutições para o mesmo volume, a duração da atividade eletromiográfica foi calculada através da diferença entre o final da última deglutição e o início da primeira deglutição.

Os dados do registro eram salvos em arquivo texto para que pudessem ser lidos em um programa especialmente desenvolvido em Matlab para análise dos sinais. O programa do Matlab retira o offset do sinal, retifica, calcula a amplitude RMS em uma janela móvel de 100 pontos e plota os sinais (Figura 2).

Esta pesquisa foi aprovada pelo Comitê de Ética em Pesquisa (CEP) com seres humanos do Centro de Ciências da Saúde da Universidade Federal de Pernambuco (CCS/UFPE) pelo Of. № 334/2008.

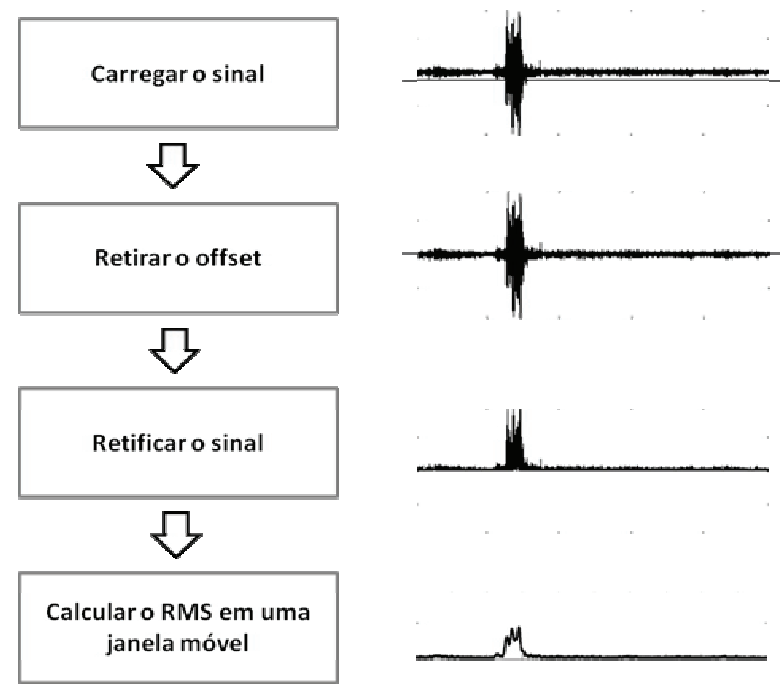

Figura 2 - Processamento e análise do sinal da EMGs através de um programa desenvolvido em Matlab 


\section{RESULTADOS}

O sujeito normal $(\mathrm{N})$ realizou uma deglutição de uma só vez de $20 \mathrm{ml}$ de água, enquanto o sujeito com DP precisou de duas deglutições para a mesma condição. Na deglutição livre de $100 \mathrm{ml}$ de água o sujeito normal realizou 6 deglutições na janela de 10 segundos, enquanto o sujeito com DP realizou 4 deglutições (6 vs 4) na mesma condição (Figura 3).

No sujeito com DP foi necessário um tempo maior do que 10 segundos para deglutição completa dos $100 \mathrm{ml}$ livre de água. Observa-se ainda um intervalo de tempo maior entre as 4 deglutições realizadas pelo sujeito com DP na condição de 100 $\mathrm{ml}$ livre de água na janela de 10 segundos em relação ao sujeito normal.

A média da amplitude ${ }_{\text {rms }}$ do sujeito normal foi maior em todos os canais do que a média da ampli- tude $_{\text {rms }}$ das duas deglutições do sujeito DP. Essa característica foi observada na deglutição de uma só vez dos volumes de $10 \mathrm{ml}$ e $20 \mathrm{ml}$ de água. A duração da atividade eletromiográfica do sujeito normal foi menor em todos os canais do que a duração das duas deglutições realizadas pelo sujeito com DP na deglutição de uma só vez de $10 \mathrm{ml}$ e 20 $\mathrm{ml}$ de água (Tabela 1).

Nos volumes de $5 \mathrm{ml} \mathrm{e} 10 \mathrm{ml}$ de iogurte consistente, em ambos os sujeitos, houve apenas uma deglutição.

A duração da atividade eletromiográfica na deglutição de uma só vez de $5 \mathrm{ml} \mathrm{e} 10 \mathrm{ml}$ de iogurte consistente no sujeito normal foi menor do que a duração da deglutição do sujeito com DP na mesma condição (Tabela 2). Ambos os sujeitos apresentaram apenas uma deglutição de uma só vez para os volumes de $5 \mathrm{ml} \mathrm{e} 10 \mathrm{ml}$ de iogurte consistente.
$\mathbf{N}$

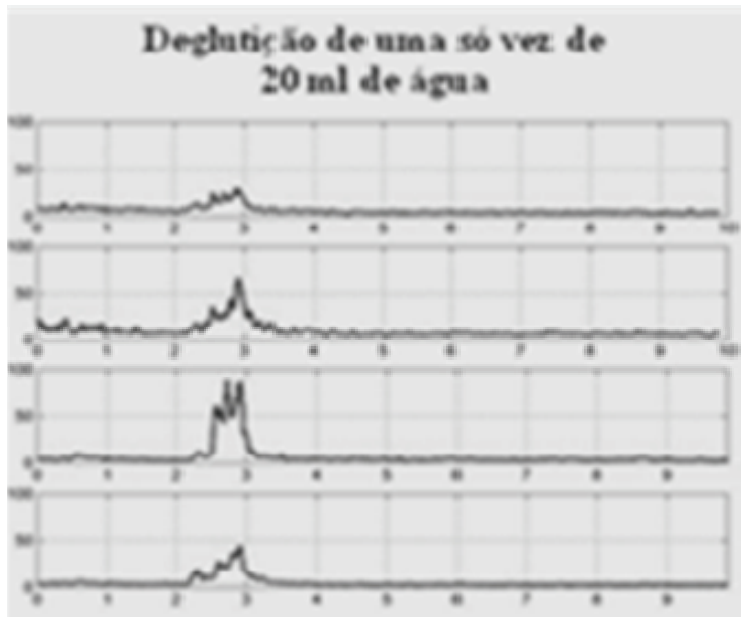

$\mathbf{P}$

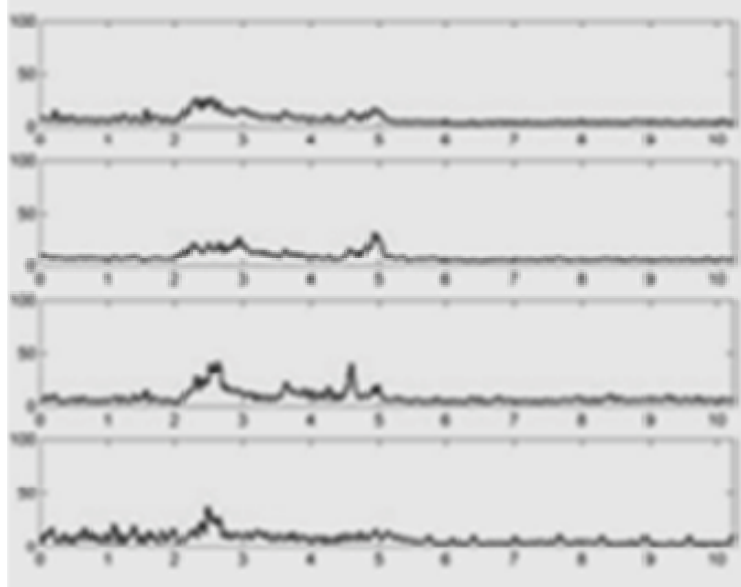

Declutic to livre de $100 \mathrm{inl}$ de agua
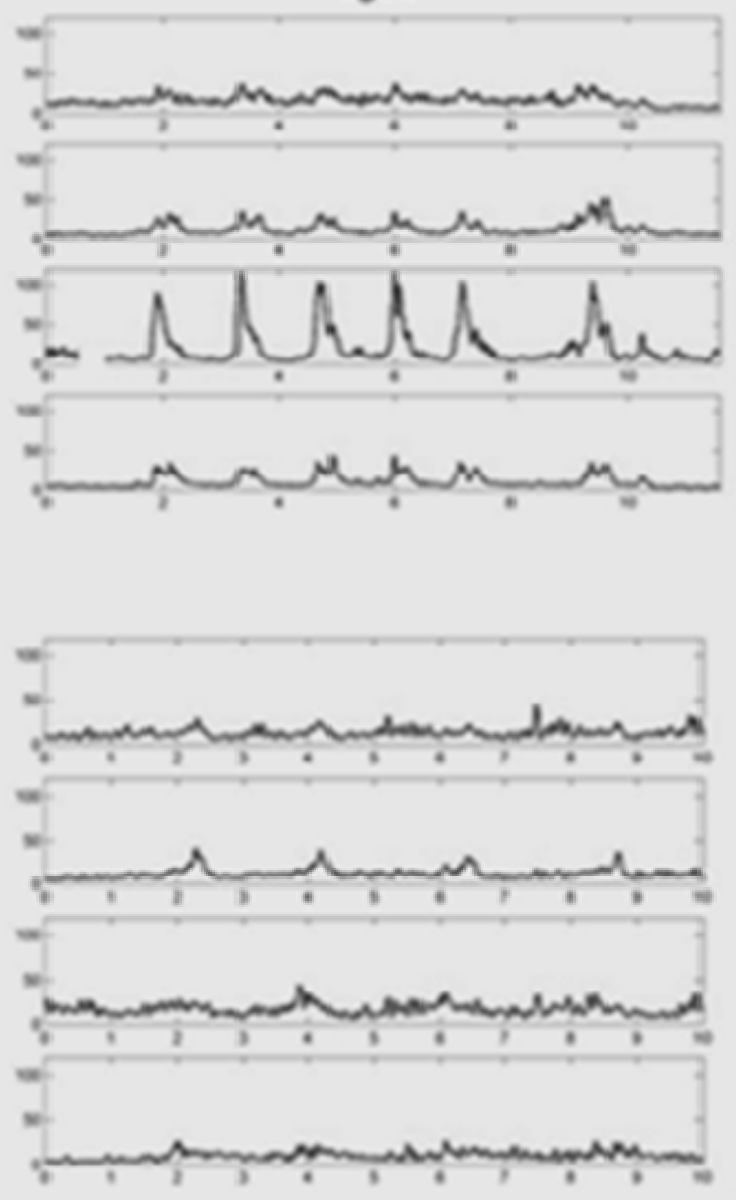

$\mathrm{N}=$ Normal e $\mathrm{P}=$ doença de Parkinson

Figura 3 - Amplitude(rms) dos sujeitos 
Tabela 1 - Deglutição de 10 e $20 \mathrm{ml}$ de água

\begin{tabular}{ccccccccc}
\hline & \multicolumn{2}{c}{ Orbicular } & \multicolumn{2}{c}{ Masséter } & \multicolumn{2}{c}{ Supra-Hióide } & \multicolumn{2}{c}{ Infra-Hióide } \\
\cline { 2 - 9 } & $\mathbf{1 0} \mathbf{~ m l}$ & $\mathbf{2 0} \mathbf{~ m l}$ & $\mathbf{1 0} \mathbf{~} \mathbf{2 0}$ & $\mathbf{~ m l}$ & $\mathbf{1 0} \mathbf{~ m l}$ & $\mathbf{2 0} \mathbf{~ m l}$ & $\mathbf{1 0} \mathbf{~} \mathbf{~}$ & $\mathbf{2 0} \mathbf{~ m l}$ \\
\hline $\mathrm{N}$ & 15,68 & 15,51 & 23,85 & 27,66 & 25,12 & 40,76 & 14,25 & 14,91 \\
$\mathrm{DP}$ & 10,3 & 10,8 & 15,1 & 18,8 & 16,5 & 21,9 & 11,5 & 10,29 \\
\hline $\mathrm{N}$ & 1,6 & 1,04 & 2,35 & 1,09 & 1,36 & 1,05 & 1,57 & 1,58 \\
$\mathrm{DP}$ & 5 & 3,8 & 4,9 & 2,4 & 4,8 & 2,5 & 5,4 & 9,6 \\
\hline
\end{tabular}

Média da amplitude ${ }_{(\mathrm{ms})}$ em milivolts (linhas superiores) e duração em segundos (linhas inferiores)

Tabela 2 - Deglutição de 5 e 10 ML de iogurte consistente

\begin{tabular}{ccccccccc}
\hline & \multicolumn{2}{c}{ Orbicular } & \multicolumn{2}{c}{ Masséter } & \multicolumn{2}{c}{ Supra-Hióide } & \multicolumn{2}{c}{ Infra-Hióide } \\
\cline { 2 - 9 } & $\mathbf{1 0} \mathbf{~ m l}$ & $\mathbf{2 0} \mathbf{~} \mathbf{l}$ & $\mathbf{1 0} \mathbf{~} \mathbf{2 0}$ & $\mathbf{2 0} \mathbf{~ l}$ & $\mathbf{1 0} \mathbf{~ m l}$ & $\mathbf{2 0} \mathbf{~ m l}$ & $\mathbf{1 0} \mathbf{~} \mathbf{~}$ & $\mathbf{2 0} \mathbf{~ m l}$ \\
\hline $\mathrm{N}$ & 15,85 & 15,46 & 28,62 & 24,09 & 39,63 & 33,19 & 17,02 & 16,38 \\
$\mathrm{DP}$ & 23,4 & 18,9 & 15,1 & 15,3 & 23,4 & 18 & 9,8 & 8,9 \\
\hline $\mathrm{N}$ & 1,7 & 2,76 & 1,51 & 3,33 & 1,23 & 2,45 & 1,68 & 2,42 \\
$\mathrm{DP}$ & 6,69 & 10 & 5,45 & 9 & 4,88 & 9,8 & 3,4 & 10,1 \\
\hline
\end{tabular}

Média da amplitude ${ }_{(\mathrm{ms})}$ em milivolts (linhas superiores) e duração em segundos (linhas inferiores)

\section{DISCUSSÃO}

Para monitorar a deglutição, principalmente quando há suspeita de disfagia, existem diversos métodos recomendados como os que utilizam esofagografia, videofluoroscopia, endoscopia, ultrassonografia, manometria, eletromiografia, cintilografia e monitorização do $\mathrm{pH}$ esofágico ${ }^{1,14}$.

Dentre esses exames instrumentais utilizados na avaliação da disfagia, a videofluoroscopia é considerada o método-ouro na investigação de sua natureza e extensão ${ }^{5,6}$. Entretanto falta a este método a mesma definição temporal que o estudo eletrofisiológico proporciona para avaliar o sincronismo e o padrão de cada evento funcional da deglutição ${ }^{13}$.

A eletromiografia de superfície é um exame complementar importante para monitorar a deglutição. Entretanto não substitui os demais exames. Envolve a detecção e o registro de potenciais elétricos das fibras musculares esqueléticas. Esse registro requer um sistema de três fases: uma fase de entrada, que inclui os eletrodos para captar os potenciais elétricos do músculo em contração; uma fase de processamento, durante a qual o pequeno sinal elétrico é amplificado; e uma fase de saída, na qual o sinal elétrico é convertido em sinais visuais e/ ou auditivos, de modo que possam ser visualizados e analisados ${ }^{15}$.

Este potencial elétrico da fibra muscular ou potencial de ação muscular composto (PAMC) é o somatório dos potenciais de ação gerados pelas fibras musculares, captados por eletrodos colocados sobre a pele que recobre o músculo ${ }^{16}$.

A precisão na descrição do método de registro eletromiográfico, das especificações técnicas do equipamento e do processamento e análise do sinal são fundamentais para a reprodutibilidade do exame e confiabilidade dos resultados.

A investigação eletrofisiológica dos músculos envolvidos na deglutição tem sido adotada em alguns trabalhos utilizando a eletromiografia de superfície para quantificar determinados parâmetros e estabelecer padrões na deglutição normal e na disfagia $7,8,10,13$.

Através do protocolo proposto foi possível demonstrar a deglutição de um sujeito normal e de um sujeito com DP através da comparação e identificação das diferenças nos parâmetros eletrofisiológicos nesses sujeitos.

Os parâmetros eletrofisiológicos avaliados através da EMGs são: a duração da atividade eletromiográfica, a amplitude ${ }_{\text {rms }}$ e o limite de disfagia. Esses dados refletem o tempo total gasto para a deglutição de um determinado volume, a intensidade da contração muscular para a deglutição de um determinado volume e a quantidade de deglutições necessárias para um determinado volume ${ }^{8,13}$.

A duração da atividade eletromiográfica caracteriza a duração da fase orofaríngea da deglutição ${ }^{12} \mathrm{e}$ o tempo que o sujeito precisa para engolir determinado volume ${ }^{7}$. Em sujeitos com DP este parâmetro encontra-se aumentado ${ }^{2,10}$, achado esse confirmado em nosso estudo em ambas as consistências. 
A amplitude ${ }_{\text {ms }}$ pode estabelecer uma relação entre a atividade elétrica e a força muscular ${ }^{8}$. Nos nossos resultados, a amplitude ${ }_{\text {rms }}$ não apresentou diferença significativa entre os sujeitos DP e normais. Esse achado foi encontrado também nos estudos de Belo $L R$ et $A L{ }^{17}$ e de Ertekin $C$ et $A L{ }^{13}$. Na maioria dos estudos o parâmetro eletrofisiológico mais importante na avaliação da deglutição é a duração da atividade eletromiográfica ${ }^{8,10,13,17}$.

Já o limite de disfagia é um parâmetro importante que caracteriza a disfagia e estabelece que o sujeito sem disfagia é capaz de realizar uma deglutição de $20 \mathrm{ml}$ de água de uma vez só $7,8,10$.

Apesar dos resultados, confirmarem os achados descritos na literatura, principalmente no que se refere à duração da atividade eletromiográfica durante a deglutição, vale ressaltar a importância da realização de outros estudos que utilizem o método descrito e o protocolo proposto numa amostra representativa que compare a deglutição de sujeitos normais e com DP que possa oferecer poder de generalização em relação às variáveis amplitude e duração da atividade eletromiográfica.

\section{CONCLUSÕES}

A eletromiografia de superfície é um método simples de monitorização da deglutição de sujeitos normais ou com DP, apresenta um custo reduzido, é reprodutível e proporciona mínimo desconforto para o examinado.

\begin{abstract}
Purpose: to describe the registering method of the surface electromyography (sEMG) through the use of a protocol developed for swallowing study and to demonstrate the swallowing pattern of a patient with Parkinson's disease and of normal individuals through the sEMG registering. Methods: to illustrate the registered electrophysiologic parameters execution of the protocol we used two volunteers of the feminine gender, being one without disease and the other one with Parkinson's disease (PD) in III period of training in accordance with the scale of Hoehn and Yahr. The analyzed parameters had been the duration of the electric activity during swallowing, the amplitude (rms) and the dysphagia limit. Results: the results show differences amongst the illustrative electromyograms. Conclusion: sEMG can be used as method for evaluating and monitoring the swallowing pattern of citizens with no disease and with PD.
\end{abstract}

KEYWORDS: Deglutition; Deglutition Disorders; Neurophysiology; Electromyography

\section{REFERÊNCIAS}

1. Dray TG, Hillel AD, Miller RM. Dysphagia caused by neurologic deficits. Otolaryngol Clin North Am. 1998; 31(3):507-24.

2. Ertekin C, Aydogdu I, YüceyarN, Tarlaci S, Kiylioglu $\mathrm{N}$, Pehlivan M, et al. Electrodiagnostic methods for neurogenic dysphagia. Electroencephalogr Clin Neurophysiol. 1998; 109(4):331-40.

3. Steenhagen CHVA, Motta LB. Deglutição e envelhecimento: enfoque nas manobras facilitadoras e posturais utilizadas na reabilitação do paciente disfágico. Rev Bras Geriatr Gerontol. 2006; 9(3):89-100.

4. Ertekin $C$, Pehlivan $M$, Aydogdu I, ErtasI M, Uludag B, Çlelebi G, et al. An electrophysiological investigation of deglutition in man. Muscle Nerve. 1995; 18(10):1177-86. http://dx.doi.org/10.1002/ mus. 880181014
5. Bigal A, Harumi D, Luz M, De Luccia G, Bilton T. Disfagia do idoso: estudo videofluoroscópico de idosos com e sem doença de Parkinson. Dist Comun. 2007; 19(2):213-23.

6. Marques CHD, André C, Rosso ALZ. Disfagia no AVE agudo: revisão sistemática sobre métodos de avaliação. Acta Fisiatr. 2008; 15(2):106-10.

7. Alfonsi E, Versino M, Merlo IM, Pacchetti C, Martignoni E, Bertino G, et al. Electrophysiologic patterns of oral-pharyngeal swallowing in parkinsonian syndromes. Neurology. 2007; 68(8):583-9.

8. Vaiman M, Eviatar E, Segal S. Surface electromyographic studies of swallowing in normal subjects: a review of 440 adults. Report 1 . Quantitative data: Timing measures. Otolaryngol Head Neck Surg. 2004; 131(4):548-55. http://dx.doi. org/10.1016/j.otohns.2004.03.013 
9. Sugita $\mathrm{K}$, Inoue M, Taniguchi H, Ootaki S, Igarashi A, Yamada Y. Effects of food consistency on tongue pressure during swallowing. J Oral Biosci. 2006; 48(4):278-85. http://dx.doi.org/10.2330/ joralbiosci/48.278

10. Potulska A, Friedman AA, Królicki L, Spychala A. Swallowing disorders in Parkinson's disease. Parkinson Related Disord. 2003; 9(6):349-53.

11. Wheeler KM, Chiara T, Sapienza CM. Surface electromyographic activity of the submental muscles during swallow and expiratory pressure threshold training tasks. Dysphagia. 2007; 22(2):108-16.

12. Gross RD, Atwood Jr CW, Ross SB, Eichhorn $\mathrm{KA}$, Olszewski JW, Doyle PJ. The coordination of breathing and swallowing in Parkinson's disease. Dysphagia. 2008; 23(2):136-45.

13. Ertekin C, Tarlaci S, Aydogdu I, Yüceyar N, Turman B, Secil Y, et al. Electrophysiological evaluation of pharyngeal phase of swallowing in patients with Parkinson's disease. Mov Disord. 2002; 17(5):942-9. http://dx.doi.org/10.1002/mds. 10240 14. Olszewski J. [Causes, diagnosis and treatment of neurogenic dysphagia as an interdisciplinary clinical problem]. Otolaryngol Pol. 2006; 60(4):491-500.

15. Portney L. Eletromiografia e testes de velocidade de condução nervosa. In: O'Sullivan SB, Schmitz TJ. Fisioterapia: avaliação e tratamento. São Paulo: Manole; 1993.p. 183-217.

16. Fowler CJ. Clinical measurement of nerve condition. Oxford: J.W. Osselton; 1995.p. 60-75.

17. Belo LR, Lins OG, Cunha DA, Lins O, Amorim CF. Eletromiografia de superfície da musculatura supra-hióidea durante a deglutição de idosos sem doenças neurológicas e idosos com Parkinson. Rev. CEFAC. 2009; 11(2):268-80. http://dx.doi. org/10.1590/S1516-18462009000200012

RECEBIDO EM: 02/09/2009

ACEITO EM: 30/11/2009

Endereço para correspondência:

Maria das Graças Wanderley de Sales Coriolano

Rua Jerônymo Vilela 665B

Recife - PE

CEP: $52040-180$

E-mail: gracawander@ hotmail.com 